\title{
A FIXED POINT THEOREM
}

\section{ELDON DYER}

Suppose that space is metric. A chain is a finite collection of open sets $d_{1}, d_{2}, \cdots, d_{n}$ such that $d_{i}$ intersects $d_{j}$ if and only if $|i-j| \leqq 1$. If the elements of a chain are of diameter less than a positive number $\epsilon$, that chain is said to be an $\epsilon$-chain. A compact continuum is said to be chainable if for each positive number $\epsilon$, there is an $\epsilon$-chain covering it. R. H. Bing has called [1] such continua snake-like. In $1951 \mathrm{O}$. H. Hamilton showed [4] that every compact chainable continuum has the fixed point property; i.e., that if $f$ is a continuous mapping of such a continuum $M$ into itself, then some point of $M$ is its own image under $f$.

In the present paper it is shown that the Cartesian product of finitely many compact chainable continua has the fixed point property. Since arcs are compact chainable continua, this is a generalization of the Brouwer fixed point theorem. Two other examples of compact chainable continua are the closure of the graph of $\sin (1 / x)$, $0<x \leqq 1$, and the pseudo-arc. Other examples are given in [1].

After reading the original manuscript of this paper, A. D. Wallace raised the question as to whether the Cartesian product of finitely many compact chainable continua is a quasi-complex (p. 323 of [6]). Rather surprisingly, the answer to this question is in the affirmative. A proof of this theorem is also given here. Since the Cartesian product of finitely many compact chainable continua is zero-cyclic, the fact that such products have the fixed point property is a special case of the Lefschetz fixed point theorem for zero-cyclic quasi-complexes. Since the author's original argument is of a very different nature, it is also given.

Let $E^{n}$ denote Euclidean $n$-space and $R^{n}$ the set of all points of $E^{n}$ whose distance from the origin is not greater than one. Let $S^{n-1}$ denote the set of all points of $E^{n}$ whose distance from the origin is one. Let $I$ denote the set of all points on the $x$-axis having abscissa $x$ such that $0 \leqq x \leqq 1$, and let $I^{n}$ denote the set of all points of $E^{n}$ each of whose $n$ coordinates $x_{i}$ satisfies $0 \leqq x_{i} \leqq 1$. If $P$ is a point of $E^{n}$ having coordinates $\left(x_{1}, x_{2}, \cdots, x_{n}\right)$ and $t$ is a real number, by $t P$ is meant the point of $E^{n}$ having coordinates $\left(t x_{1}, t x_{2}, \cdots, t x_{n}\right)$.

Presented to the Society, April 15, 1955; received by the editors February 23, 1955, and, in revised form, July 2, 1955. 
For definitions of those concepts from homotopy theory which are used in this paper the reader is referred to $\$ 3$ of Chapter VI of [5].

LEMma 1. Suppose $f$ and $g$ are continuous mappings of $R^{n}$ into itself and $g \mid S^{n-1}$ is an essential mapping onto $S^{n-1}$. Then there is a point $x$ in $R^{n}$ such that $f(x)=g(x)$.

Proof. Suppose there is no point $x$ in $R^{n}$ such that $f(x)=g(x)$. For each point $x$ in $R^{n}$ let $h(x)=\circ(x)-f(x)$. For each point $x$ in $S^{n-1}$ and number $t, 0 \leqq t \leqq 1$, let $H(x, t)=h(t x) /|h(t x)|$. This is possible since $h(x) \neq 0$ for any $x$ in $R^{n} . H(x, t)$ is continuous in $x$ and $t$.

If $H(x, 1)$ and $g(x)$ were not antipodal for any point $x$ in $S^{n-1}$, they would be homotopic; but $g \mid S^{n-1}$ is essential and $H\left(S^{n-1}, 1\right)$ is homotopic to the constant map $H\left(S^{n-1}, 0\right)$. Hence, there is a point $x$ in $S^{n-1}$ such that $H(x, 1)=-g(x)$. Then $g(x) \cdot(1+|g(x)-f(x)|)=f(x)$. Let $k=1+|g(x)-f(x)| \cdot k \cdot g(x)=f(x)$. Since $|g(x)|=1$ and $|f(x)| \leqq 1$, $k \leqq 1$; but by its definition $k \geqq 1$. Therefore, $g(x)=f(x)$.

Lemma 2. Suppose that for each integer $i, 1 \leqq i \leqq n, f_{i}$ is a continuous mapping of $I$ onto $I$ such that $f_{i}(0)=0$ and $f_{i}(1)=1$. For each point $x=\left(x_{1}, x_{2}, \cdots, x_{n}\right)$ of $I^{n}$ let $f(x)=\left(f_{1}\left(x_{1}\right), f_{2}\left(x_{2}\right), \cdots, f_{n}\left(x_{n}\right)\right)$. Let $T^{n-1}$ denote the set of all points $x$ of $I^{n}$ such that for some $i, x_{i}$ is either 0 or 1 . Then $f\left(T^{n-1}\right)=T^{n-1}$, and $f \mid T^{n-1}$ is homotopic to the identity map of $T^{n-1}$ onto itself.

Proof. For each integer $i, 1 \leqq i \leqq n$, there is a continuous mapping $h_{i}(x, t)$ of $I \times I$ onto $I$ such that $h_{i}(x, 0)=f_{i}(x), h_{i}(x, 1)=x$, and $h_{i}(0, t)=0$ and $h_{i}(1, t)=1$ for $0 \leqq t \leqq 1$. Let $H(x, t), 0 \leqq t \leqq 1$ and $x$ in $I^{n}$, be defined as follows: $H(x, t)=\left(h_{1}\left(x_{1}, 1\right), \cdots, h_{i-1}\left(x_{i-1}, 1\right)\right.$, $\left.h_{i}\left(x_{i}, u\right), h_{i+1}\left(x_{i+1}, 0\right), \cdots, h_{n}\left(x_{n}, 0\right)\right)$, where $(i-1) / n \leqq t \leqq i / n$ and $u=n \cdot[t-(i-1) / n]$. It can easily be shown that $H(x, t)$ is continuous in $x$ and $t$, that $H(x, 0)=f(x)$ for all $x$ in $I^{n}$, that $H(x, 1)=x$ for all $x$ in $T^{n-1}$ (in fact in $I^{n}$ ), and that $H\left(T^{n-1}, t\right)=T^{n-1}$ for all $t$, $0 \leqq t \leqq 1$.

Theorem 1. Suppose that $M$ is the Cartesian product of $n$ compact chainable continua $X_{1}, X_{2}, \cdots, X_{n}$ and $f$ is a continuous mapping of $M$ into itself. Then there is a point $x$ of $M$ such that $x=f(x)$.

Proof. Let $M$ be all of space. Suppose there is no point $x$ of $M$ such that $x=f(x)$. There is a positive number $e$ such that $d(x, f(x))$ $>4 n e$ for all points $x$ in $\dot{M}$. For each integer $i, 1 \leqq i \leqq n$, there is a chain $A_{i}$ covering $X_{i}$ such that each element of the collection $C_{a}$ of all ordered $n$-tuples $\left(a_{1}, a_{2}, \cdots, a_{n}\right)$, where $a_{i}$ is in $A_{i}$, has diameter less than $e$. For each integer $i, 1 \leqq i \leqq n$, there is a chain $B_{i}^{\prime}$ covering 
$X_{i}$ such that $B_{i}^{\prime}$ is a refinement of $A_{i}$ and each element of the collection $C_{b}^{\prime}$ of all ordered $n$-tuples $\left(b_{1}, b_{2}, \cdots, b_{n}\right)$, where $b_{i}$ is in $B_{i}^{\prime}$, is mapped by $f$ into some element of $C_{a}$. For each $i$ let $B_{i}$ denote a subchain of $B_{i}^{\prime}$ which is irreducible with respect to covering points in the first and last links of $A_{i}$ which are not in any other link of $A_{i}$, and let $C_{b}$ denote the subcollection of $C_{b}^{\prime}$ of all ordered $n$-tuples $\left(b_{1}, b_{2}, \cdots, b_{n}\right)$, where $b_{i}$ is in $B_{i}$. For each $i$ let $\alpha_{i}$ denote the number of links of $A_{i}$ and $\beta_{i}$ denote the number of links of $B_{i}$. If for some $i$, the first link of $B_{i}$ lies in the last link of $A_{i}$, renumber the links of $B_{i}$ so that its $j$ th link becomes its $\left(\beta_{i}-j+1\right)$ st link.

For each $x=\left(x_{1}, x_{2}, \cdots, x_{n}\right)$, where $x_{i}=k_{i} /\left(\beta_{i}-1\right), k_{i}$ being an integer such that $0 \leqq k_{i} \leqq \beta_{i}-1$, let $\rho_{x}$ be the element of $C_{b}$ whose $i$ th term is the $\left(k_{i}+1\right)$ st link in $B_{i} . f\left(\rho_{x}\right)$ is in some element $\theta$ of $C_{a}$. The $i$ th term of $\theta$ is the $p_{i}$ th link of $A_{i}$. There are two adjacent positive integers $m_{i}$ and $m_{i}+1$ such that the $i$ th term of any element of $C_{a}$ containing $f\left(\rho_{x}\right)$ is either the $m_{i}$ th or the $\left(m_{i}+1\right)$ st link of $A_{i}$ and one of the numbers $m_{i}$ and $m_{i}+1$ is $p_{i}$. If no element of $C_{a}$ containing $f\left(\rho_{x}\right)$ has an $i$ th term other than the $p_{i}$ th link of $A_{i}$, let

$$
f_{i}(x)=\left(p_{i}-1\right) /\left(\alpha_{i}-1\right) .
$$

If some element of $C_{a}$ containing $f\left(\rho_{x}\right)$ has an $i$ th term other than the $p_{i}$ th link of $A_{i}$, let $f_{i}(x)=\left(2 m_{i}-1\right) / 2\left(\alpha_{i}-1\right)$. Let

$$
F(x)=\left(f_{1}(x), f_{2}(x), \cdots, f_{n}(x)\right) .
$$

Let $B$ denote the set of all points $x=\left(x_{1}, x_{2}, \cdots, x_{n}\right)$ of $I^{n}$ such that for each integer $i, 1 \leqq i \leqq n$, there is an integer $k_{i}, 0 \leqq k_{i} \leqq \beta_{i}-1$, such that $x_{i}=k_{i} /\left(\beta_{i}-1\right)$. Let $\mathfrak{B}$ denote the collection of all sets $\sigma$ of $2^{n}$ points of $B$ for which there is a point $P$ of $\sigma$ such that for every point $Q$ of $\sigma$ and for each integer $i, 1 \leqq i \leqq n$, the $i$ th coordinate of $Q$ either equals the $i$ th coordinate of $P$ or exceeds it by 1 . It can be shown (see, for example, $\S 8$ of Chapter II of [3]) that there is an $n$-complex $\beta$ which is a triangulation of $I^{n}$ such that the vertex set of each simplex in $\beta$ is a subset of an element of $\mathfrak{B}$.

For each point $x$ in $I^{n}$ and simplex $\sigma$ of $\beta$ containing it, let $\left(a_{0}, a_{1}, \cdots, a_{j}\right)$ be the barycentric coordinates of $x$ with respect to the vertex set $\left(s_{0}, s_{1}, \cdots, s_{j}\right)$ of $\sigma . x=a_{0} s_{0}+a_{1} s_{1}+\cdots+a_{j} s_{j}$. Define $F_{\sigma}(x)$ to be $a_{0} F\left(s_{0}\right)+a_{1} F\left(s_{1}\right)+\cdots+a_{j} F\left(s_{j}\right)$. Clearly, $F_{\sigma}$ is continuous on $\sigma$. If $x$ is common to two simplexes $\sigma_{1}$ and $\sigma_{2}$ of $\beta$, let $\sigma$ denote their common face. Then $F_{\sigma_{1}}(x)=F_{\sigma}(x)=F_{\sigma_{2}}(x)$. Hence, if $F(x)$ denotes $F_{\sigma}(x)$ for any simplex $\sigma$ of $\beta$ containing $x, F$ is a continuous mapping of $I^{n}$ into itself. Furthermore, if $P$ is a vertex of a simplex of $\beta$ containing the point $x$, for each integer $i, 1 \leqq i \leqq n$, the 
$i$ th coordinates of $F(P)$ and $F(x)$ do not differ by more than $1 /\left(\alpha_{i}-1\right)$. Let $g_{i}\left((k-1) /\left(\beta_{i}-1\right)\right)=(j-1) /\left(\alpha_{i}-1\right)$ if the $k$ th link of $B_{i}$ lies in only the $j$ th link of $A_{i}$. If the $k$ th link of $B_{i}$ lies in two links of $A_{i}$, the $j$ th and $(j+1)$ st, let $g_{i}\left((k-1) /\left(\beta_{i}-1\right)\right)=(2 j-1) / 2\left(\alpha_{i}-1\right)$. Let $g_{i}(I)=I$ be the piecewise linear extension of this mapping. For each point $x$ of $I$ such that $(k-1) /\left(\beta_{i}-1\right) \leqq x \leqq k /\left(\beta_{i}-1\right), g_{i}(x)$ is between $g_{i}\left((k-1) /\left(\beta_{i}-1\right)\right)$ and $g_{i}\left(k /\left(\beta_{i}-1\right)\right)$. For each point $x$ in $I^{n}$, let $g(x)=\left(g_{1}\left(x_{1}\right), g_{2}\left(x_{2}\right), \cdots, g_{n}\left(x_{n}\right)\right)$. Let $T^{n-1}$ denote the boundary of $I^{n}$. By Lemma $2, g \mid T^{n-1}$ is homotopic to the identity mapping of $T^{n-1}$ onto itself; hence, $g \mid T^{n-1}$ is essential onto $T^{n-1}$.

By Lemma 1 , there is a point $x$ of $I^{n}$ such that $F(x)=g(x)$. Let $v$ denote a vertex of a simplex of $\beta$ containing $x$. For each integer $i$, $1 \leqq i \leqq n$, the $i$ th coordinate of $F(x)$ does not differ from the $i$ th coordinate of $F(v)$ by more than $1 /\left(\alpha_{i}-1\right)$. Also, the $i$ th coordinate of $g(x)$ does not differ from the $i$ th coordinate of $g(v)$ by more than $1 /\left(\alpha_{i}-1\right)$. Hence, the $i$ th coordinate of $F(v)$ does not differ from the $i$ th coordinate of $g(v)$ by more than $2 /\left(\alpha_{i}-1\right)$. The point $v$ is an element of the set $B$; i.e., $v=\left(v_{1}, v_{2}, \cdots, v_{n}\right)$, where for each integer $i$, $1 \leqq i \leqq n$, there is an integer $k_{i}, 0 \leqq k_{i} \leqq \beta_{i}-1$, such that $v_{i}=k_{i} /\left(\beta_{i}-1\right)$. Let $V$ denote the element of the set $C_{b}$ whose $i$ th term is the $\left(k_{i}+1\right)$ st link of $B_{i}$. Let \& denote an element of $C_{a}$ such that for each $i$, the $i$ th term of $V$ lies in the $i$ th term of $(\$)$ and let $\mathfrak{F}$ be an element of $C_{a}$ containing $f(V)$. For each $i$ there is a subchain in $A_{i}$ from the $i$ th term of $\$$ to the $i$ th term of $\mathfrak{F}$ having not more than 4 links. Therefore, there is a chain in $C_{a}$ from $(5)$ to $\mathfrak{F}$ having not more than $4 n$ links. Each of these links is of diameter less than $e$; therefore, $d(V, f(V))$ $<4 n e$. This is a contradiction.

Corollary. The Cartesian product of the elements of any collection of compact chainable continua has the fixed point property.

Proof. This follows immediately from Theorem 1 and the theorem that if $G$ is a collection of compact Hausdorff spaces such that the Cartesian product of the elements of each finite subcollection of $G$ has the fixed point property, then the Cartesian product of the elements of the collection $G$ has the fixed point property.

For the definition of the term quasi-complex the reader is referred to p. 323 of [6]. Notation and terminology used, with only a few exceptions, are in conformity with that used in [6].

LEMMA 3. If $\alpha$ and $\beta$ are arc-like finite simplicial complexes and $\pi$ is a simplicial mapping of $\beta$ onto $\alpha$, there exists a chain mapping $\omega$ of $\alpha$ into $\beta$ such that

(i) for each zero-chain $\gamma^{0}$ of $\alpha, K I\left(\gamma^{0}\right)=K I\left(\omega\left(\gamma^{0}\right)\right)$; 
(ii) for each $i$-simplex $\sigma^{i}$ of $\alpha$ and $i$-simplex $\gamma^{i}$ of $\beta$ having nonzero coefficient in $\omega\left(1 \cdot \sigma^{i}\right), \pi\left(\gamma^{i}\right) \subset \sigma^{i}$; and (iii) $\omega \pi \sim 1$.

Proof. Let $a_{1}, a_{2}, \cdots, a_{n}$ denote the vertices of $\alpha$ ordered as on $\alpha$. There is a subarc $\beta^{\prime}$ of $\beta$ such that $\pi\left(\beta^{\prime}\right)=\alpha$ and there is no proper subarc $\gamma$ of $\beta^{\prime}$ such that $\pi(\gamma)=\alpha$. Let $b_{1}$ denote the vertex of $\beta^{\prime}$ such that $\pi\left(b_{1}\right)=a_{1}$ and let $b_{1}, b_{2}, \cdots, b_{m}$ denote the vertices of $\beta^{\prime}$ ordered as on $\beta^{\prime}$. There is a subsequence $b_{k_{1}}, b_{k_{2}}, \cdots, b_{k_{p}}$ of $b_{1}, b_{2}, \cdots, b_{m}$ such that

(1) $\pi\left(b_{k_{1}}\right)=a_{1}$ and $\pi\left(b_{k_{p}}\right)=a_{n}$;

(2) if $\pi\left(b_{k_{i}}\right)=a_{j}$ and $\pi\left(b_{k_{i+1}}\right)=a_{k}$, then $|j-k| \leqq 1$; and

(3) for each $i, k_{i+1}$ is the greatest integer $j$ such that

(a) $k_{i} \leqq j \leqq k_{p}$ and

(b) if $k_{i}<q \leqq j, \pi\left(b_{q}\right) \in\left\{\pi\left(b_{k_{i}}\right)\right\} \cup\left\{\pi\left(b_{k_{i}+1}\right)\right\}$.

Define $\omega\left(1 \cdot a_{i}\right)$ to be $\sum_{j=1}^{p} X_{i}^{j} \cdot b_{k_{j}}$, where $X_{i}^{j}=0$ if $\pi\left(b_{k_{j}}\right) \neq a_{i}$,

and if $\pi\left(b_{k_{j}}\right)=a_{i}$ and $\left\{\begin{aligned} \pi\left(b_{k_{j}+1}\right)=a_{i+1}, & \text { then } X_{i}^{j}=+1 . \\ \pi\left(b_{k_{j}+1}\right)=a_{i-1}, & \text { then } X_{i}^{j}=-1 . \\ j=p, & \text { then } X_{j}^{j}=+1 .\end{aligned}\right.$

Define $\omega\left(1 \cdot\left(a_{i}, a_{i+1}\right)\right)=\sum_{j=1}^{m-1} Y_{i}^{j} \cdot\left(b_{j}, b_{j+1}\right)$, where if $k_{s} \leqq j<j+1$ $\leqq k_{s+1}$ and for all $t, k_{s} \leqq t \leqq k_{s+1}, \pi\left(b_{t}\right) \in\left\{a_{i}\right\} \cup\left\{a_{i+1}\right\}$, then $Y_{i}^{\prime}=+1$; otherwise, $Y_{i}^{\prime}=0$.

To show that $\omega$ is a chain mapping, it will be shown that for each $j$, the coefficient of $b_{j}$ is the same in both $\omega \delta\left(1 \cdot\left(a_{i}, a_{i+1}\right)\right)$ and $\delta \omega\left(1 \cdot\left(a_{i}, a_{i+1}\right)\right)$, where $\delta$ denotes the boundary operator. Unless $j$ is some $k_{t}$, its coefficient in both expressions is zero. Suppose $j=k_{t}$.

CASE 1. $\pi\left(b_{k_{t}}\right)=a_{i+1}$. If $\pi\left(b_{k_{t}+1}\right)=a_{i+2}$, then $\left\{\pi\left(b_{k_{t-1}}\right)\right\} \cup\left\{\pi\left(b_{k_{t-1}+1}\right)\right\}$ $\left.\subset\left\{a_{i}\right\} \cup a_{i+1}\right\}$ and $\omega\left(a_{i}, a_{i+1}\right)$ contains $1 \cdot\left(b_{k-1}, b_{k_{t}}\right)$ and $0 \cdot\left(b_{k_{t}}\right.$, $\left.b_{k_{t}+1}\right)$. If $\pi\left(b_{k_{t}+1}\right)=a_{i}$, then either $\pi\left(b_{k_{t-1}}\right)$ or $\pi\left(b_{k_{t-1}+1}\right)$ does not lie in $\left\{a_{i}\right\} \cup\left\{a_{i+1}\right\}$ and $\omega\left(a_{i}, a_{i+1}\right)$ contains $0 \cdot\left(b_{k_{t}-1}, b_{k_{t}}\right)$ and $1 \cdot\left(b_{k_{t}}, b_{k_{t}+1}\right)$. Thus, $\delta \omega\left(a_{i}, a_{i+1}\right)$ contains $+1 \cdot b_{k_{t}}\left(\right.$ or $\left.-1 \cdot b_{k_{\ell}}\right)$ if $\pi\left(b_{k_{t}+1}\right)=a_{i+2}$ (or $\left.=a_{i}\right)$. This is the same coefficient for $b_{k_{t}}$ as that given by $\omega\left(1 \cdot a_{i+1}\right)$, which is the same as that given by $\omega \delta\left(a_{i}, a_{i+1}\right)$.

CASE 2. $\pi\left(b_{k_{t}}\right)=a_{i}$. If $\pi\left(b_{k_{t}+1}\right)=a_{i+1}$, then $\omega\left(a_{i}, a_{i+1}\right)$ contains $0 \cdot\left(b_{k_{t}-1}, b_{k_{t}}\right)$ and $1 \cdot\left(b_{k_{t}}, b_{k_{t}+1}\right)$. If $\pi\left(b_{k_{t}+1}\right)=a_{i-1}$, then $\omega\left(a_{i}, a_{i+1}\right)$ contains $1 \cdot\left(b_{k_{t}-1}, b_{k_{t}}\right)$ and $0 \cdot\left(b_{k_{t}}, b_{k_{t}+1}\right)$. Thus, $\delta \omega\left(a_{i}, a_{i+1}\right)$ contains $-1 \cdot b_{k_{t}}\left(\right.$ or $\left.+1 \cdot b_{k_{t}}\right)$ if $\pi\left(b_{k_{t}+1}\right)=a_{i+1}$ (or $\left.=a_{i-1}\right)$. This is the same coefficient for $b_{k_{t}}$ as that given by $\omega\left(-1 \cdot a_{i}\right)$, which is the same as that given by $\omega \delta\left(a_{i}, a_{i+1}\right)$. Thus, $\omega$ is a chain mapping.

To show that $\omega$ preserves the Kronecker index of zero-cycles, it will be shown that $\operatorname{KI}\left(\omega\left(1 \cdot a_{i}\right)\right)=1$, for each $i$. Clearly, $\operatorname{KI}\left(\omega\left(1 \cdot a_{1}\right)\right)$ 
$=\mathrm{KI}\left(\omega\left(1 \cdot a_{n}\right)\right)=1$. Suppose $1<i<n$. Let $U_{i}$ be the collection of all subarcs $\gamma$ of $\beta^{\prime}$ which are maximal with respect to $\pi(\gamma)=a_{i}$ and such that if $b_{j}, b_{j+1}, \cdots, b_{j+k}$ are the vertices of $\gamma, \pi\left(b_{j-1}\right)=a_{i-1}$ and $\pi\left(b_{j+k+1}\right)=a_{i+1}$. Let $D_{i}$ be the collection of all such maximal subarcs for which $\pi\left(b_{j-1}\right)=a_{i+1}$ and $\pi\left(b_{j+k+1}\right)=a_{i-1}$. For each element of $U_{i} \cup D_{i}$, the vertex $b_{j+k}$ is a $b_{k_{i}}$, and $\omega\left(1 \cdot a_{i}\right)$ contains $+1 \cdot b_{j+k}$ or $-1 \cdot b_{j+k}$ as $b_{j+k}$ is a vertex of an element of $U_{i}$ or $D_{i}$. Also $\omega\left(1 \cdot a_{i}\right)$ does not attach a nonzero coefficient to any $b_{k_{t}}$ which is not a vertex of some element of $U_{i} \cup D_{i}$. Thus, $\operatorname{KI}\left(\omega\left(1 \cdot a_{i}\right)\right)$ equals the number of elements of $U_{i}$ minus the number of elements of $D_{i}$. If the elements of $U_{i} \cup D_{i}$ are ordered as on $\beta^{\prime}$, between each two elements of $U_{i}$ there is an element of $D_{i}$ and between each two elements of $D_{i}$ there is an element of $U_{i}$; furthermore, the first and last elements of $U_{i} \cup D_{i}$ are in $U_{i}$. Hence, $U_{i}$ has one more element than $D_{i}$, and $\operatorname{KI}\left(\omega\left(1 \cdot a_{i}\right)\right)=1$.

For cycles $\gamma^{p}$ on $\beta$ of dimension $p=1, \omega \pi\left(\gamma^{p}\right)$ is a cycle; also $\omega \pi\left(\gamma^{p}\right)-\gamma^{p}$ is a cycle. Since $\beta$ is zero-cyclic, $\omega \pi\left(\gamma^{p}\right)-\gamma^{p} \sim 0$ or $\omega \pi\left(\gamma^{p}\right) \sim \gamma^{p}$. For any zero-cycle, $\gamma^{0}$, on $\beta, \mathrm{KI}\left(\omega \pi\left(\gamma^{0}\right)\right)=\mathrm{KI}\left(\gamma^{0}\right)$, and so $\omega \pi\left(\gamma^{0}\right) \sim \gamma^{0}$. Hence, $\psi \pi \sim 1$.

THEOREM 2. Every compact metric chainable continuum is a quasicomplex.

Proof. Let $M$ denote a compact metric chainable continuum. Let $U_{1}, U_{2}, \cdots$ be a sequence of chains covering $M$ such that $U_{i+1}$ is a refinement of $U_{i}$ and $U_{i}$ is a $(1 / i)$-chain; let $\Phi_{i}$ denote the nerve of $U_{i}$. If $i$ and $j$ are positive integers and $i<j$, let $\pi_{i}^{j}$ denote a simplicial mapping of $\Phi_{j}$ onto $\Phi_{i}$ induced by inclusion; i.e., a projection mapping. Let $\omega_{j}^{j}$ denote the chain mapping of $\Phi_{i}$ into $\Phi_{j}$ defined for $\pi_{i}^{j}$ as in Lemma 3. Antiprojections will be the mappings $\omega_{j}^{4}$ and finite products $\omega_{i_{n}^{n_{n-1}}} \cdots \omega_{t_{2}^{2}}^{i_{2}} \cdot \omega_{t_{2}}^{i_{2}}$, where $i_{1}<i_{2}<\cdots<i_{n}$. These products also preserve the Kronecker index of zero-cycles on $\Phi_{i_{1}}$. These mappings have all of the properties required in Property B on p. 323 of [6]. To show that $M$ has Property $\mathrm{C}$, for each $U_{i}(=a)$, let $U_{j}(=g)$ be a sufficiently small refinement of $U_{i}$ that any three adjacent elements of $U_{j}$ lie in some element of $U_{i}$. Then for any $U_{k}(=b)$, let $U_{m}(=h)$ be the one of the two $U_{j}$ and $U_{k}$ which is a refinement of both. Therefore, $M$ has Property C.

For two complexes $K_{1}$ and $K_{2}, K_{1} \Delta K_{2}$ denotes the simplicial product of $K_{1}$ and $K_{2}$ as defined in $\$ 8$ of Chapter II of [3].

LEMMA 4. If for each $i, 1 \leqq i \leqq n, \alpha_{i}$ is a finite simplicial complex, $\beta_{i}$ is a connected finite simplicial complex, and $\omega_{i}$ maps the zero-chains of $\alpha_{i}$ into zero-chains of $\beta_{i}$ in such a way that $\mathrm{KI}\left(\omega_{i} \delta\left(\sigma^{1}\right)\right)=0$ for each one-simplex $\sigma^{1}$ of $\alpha_{i}$, then if $\alpha=\alpha_{1} \Delta \alpha_{2} \Delta \cdots \Delta \alpha_{n}, \beta=\beta_{1} \Delta \beta_{2} \Delta \cdots$ 
$\Delta \beta_{n}$, and for each vertex $v=\left(a_{1}, a_{2}, \cdots, a_{n}\right)$ of $\alpha$, where $a_{i}$ is a vertex of $\alpha_{i}, \omega(1 \cdot v)=\sum \gamma_{b} \cdot\left(b_{1}, b_{2}, \cdots, b_{n}\right)$, where the summation extends over all vertices $b=\left(b_{1}, b_{2}, \cdots, b_{n}\right)$ of $\beta$, and for each $b, \gamma_{b}$ is the product of the coefficients of the $b_{i}$ in $\omega_{i}\left(a_{i}\right)$, then if $\gamma^{0}$ is a bounding zero-cycle in $\alpha, \omega\left(\gamma^{0}\right)$ is a bounding zero-cycle in $\beta$.

Proof. For each vertex $v$ of $\alpha$, the Kronecker index of $\omega(v)$ equals the product of the Kronecker indices of the $\omega_{i}\left(a_{i}\right)$, where $\left\{a_{i}\right\}$ are the coordinates of $v$. If $\gamma^{0}$ is the boundary of the one-simplex $\left(v_{1}, v_{2}\right)$ of $\alpha$, where all of the coordinates of $v_{1}$ and $v_{2}$ are the same except the $i$ th, then $\mathrm{KI}\left(\omega\left(\gamma^{0}\right)\right)=\mathrm{KI}\left(\omega\left(v_{2}\right)\right)-\mathrm{KI}\left(\omega\left(v_{1}\right)\right)=0$, since $\mathrm{KI}\left(\omega_{i} \delta\left(v_{1 i}, v_{2 i}\right)\right)$ $=0$. Since $\beta$ is connected, $\omega\left(\gamma^{0}\right)$ bounds. If $\gamma^{0}$ is the boundary of any one-simplex in $\alpha$, it is the sum of the boundaries of one-simplexes in $\alpha$ of the sort discussed in the previous sentence; hence, $\operatorname{KI}\left(\omega\left(\gamma^{0}\right)\right)=0$. Finally, if $\gamma^{0}=\delta \sum_{k=1}^{j} p_{k} \cdot \sigma_{k}^{1}$, where each $\sigma_{k}^{1}$ is a one-simplex of $\alpha$ and $p_{k}$ is an element of the coefficient group, $\omega\left(\gamma^{0}\right)=\omega\left(\sum_{k=1}^{j} p_{k} \cdot \delta\left(\sigma_{k}^{1}\right)\right)$ $=\sum_{k=1}^{j} p_{k} \cdot \omega \delta\left(\sigma_{k}^{1}\right)$; therefore, $\mathrm{KI}\left(\omega\left(\gamma^{0}\right)\right)=0$. Hence, in each case $\omega\left(\gamma^{0}\right)$ bounds if $\gamma^{0}$ bounds.

Lemma 5. If $\sigma$ is a complex, $\sigma^{\prime}$ is a subcomplex of $\sigma$ containing all of the vertices of $\sigma, \gamma$ is a zero-cyclic complex, and $\omega^{\prime}$ is a chain mapping of $\sigma^{\prime}$ into $\gamma$ which maps bounding zero-cycles on $\sigma^{\prime}$ into bounding zerocycles on $\gamma$, then $\omega^{\prime}$ can be extended to a chain mapping $\omega$ of $\sigma$ into $\gamma$.

Proof. Let $\alpha_{1}, \alpha_{2}, \cdots, \alpha_{k}$ denote the simplexes in $\sigma$ which are not in $\sigma^{\prime}$ ordered such that if $i<j$, then the dimension of $\alpha_{i}$ is less than or equal to the dimension of $\alpha_{j}$. The argument will be by mathematical induction. For each $i, 1 \leqq i \leqq k$, let $\sigma_{i}$ denote the subcomplex of $\sigma$ consisting of all simplexes of $\sigma^{\prime}$ and all simplexes in each of the simplexes $\alpha_{1}, \alpha_{2}, \cdots, \alpha_{i} . \omega_{1}$ is the extension of $\omega^{\prime}$ to $\sigma_{1}$ obtained as follows: since $\delta\left(\alpha_{1}\right)$ is in $\sigma^{\prime}$ and is a bounding cycle, $\omega^{\prime} \delta\left(\alpha_{1}\right)$ is a cycle in $\gamma$ which bounds in $\gamma$ if it is a zero-cycle since it is the image of a bounding zero-cycle and bounds in $\gamma$ if it is a higher-dimensional cycle since all such cycles bound in $\gamma$. Let $\tau_{1}$ denote a chain in $\gamma$ such that $\omega^{\prime} \delta\left(\alpha_{1}\right)=\delta \tau_{1}$ and let $\omega_{1}\left(\alpha_{1}\right)=\tau_{1}$. Then $\omega_{1}$ is a chain mapping of $\sigma_{1}$ into $\gamma$ and is an extension of $\omega^{\prime}$. It is clear this process can be continued inductively.

An $n$-product simplex is a simplex which is the $\Delta$ product of $n$ simplexes.

Lemмa 6. If for each $i, 1 \leqq i \leqq n, \alpha_{i}$ and $\beta_{i}$ are arc-like finite simplicial complexes and $\pi_{i}$ is a simplicial mapping of $\beta_{i}$ onto $\alpha_{i}$, then if for each vertex $b$ of $\beta=\beta_{1} \Delta \beta_{2} \Delta \cdots \Delta \beta_{n}, \pi(b)$ is the vertex $\left(\pi_{1}\left(b_{1}\right), \pi_{2}\left(b_{2}\right), \cdots\right.$, $\left.\pi_{n}\left(b_{n}\right)\right)$ of $\alpha=\alpha_{1} \Delta \alpha_{2} \Delta \cdots \Delta \alpha_{n}$, where for each $i, b_{i}$ is the ith coordinate 
of $b$, then $\pi$ is a simplicial mapping of $\beta$ onto $\alpha$ and there is a chain mapping $\omega$ of $\alpha$ into $\beta$ such that

(i) for each zero-chain $\gamma^{0}$ of $\alpha, \mathrm{KI}\left(\gamma^{0}\right)=\mathrm{KI}\left(\omega\left(\gamma^{0}\right)\right)$;

(ii) for each $k$-simplex $\sigma^{k}$ of $\alpha,\left(2^{n}-1\right)$-simplex $\sigma$ of $\alpha$ of which $\sigma^{k}$ is a face, and $k$-simplex $\gamma^{k}$ of $\beta$ having nonzero coefficient in $\omega\left(1 \cdot \sigma^{k}\right)$, $\pi\left(\gamma^{k}\right) \subset \sigma ;$ and

(iii) $\omega \pi \sim 1$.

Proof. For each vertex $v=\left(a_{1}, a_{2}, \cdots, a_{n}\right)$ of $\alpha$ and vertex $u=\left(b_{1}, b_{2}, \cdots, b_{n}\right)$ of $\beta$, let $\omega_{u}(v)=\lambda_{u} \cdot u$, where $\lambda_{u}$ is the product of the coefficients of the $b_{i}$ in $\omega_{i}\left(a_{i}\right), \omega_{i}$ being defined for $\alpha_{i}, \beta_{i}$, and $\pi_{i}$ as in Lemma 3. Let $\omega^{\prime}(v)$ be $\sum \omega_{u}(v)$, where the summation is over all vertices $u$ in $\beta . \mathrm{KI}\left(\omega^{\prime}(v)\right)$ is the product of the Kronecker indices of the $\omega_{i}\left(a_{i}\right)$ and since each of these is one, $\mathrm{KI}\left(\omega^{\prime}(v)\right)=1$.

Let $\gamma_{1}, \gamma_{2}, \cdots, \gamma_{k}$ denote the $n$-product simplexes of $\alpha$ of positive dimension ordered so that if $i<j$, then the dimension of $\gamma_{i}$ is less than or equal to the dimension of $\gamma_{j}$. For each $j$, let $\rho_{j}$ denote the subcomplex of $\alpha$ composed of all vertices of $\alpha$ and all faces of all simplexes $\gamma_{1}, \gamma_{2}, \cdots, \gamma_{j} \cdot \gamma_{1}=A_{1} \Delta A_{2} \Delta \cdots \Delta A_{n}$, where each $A_{i}$ is a simplex in $\alpha_{i}$. Let $B_{i}$ denote the collection of all maximal coherent subcomplexes of $\beta_{i}$ each simplex of which has a nonzero coefficient in $\omega_{i}\left(A_{i}\right)$. For each subcomplex $\mu=X_{1} \Delta X_{2} \Delta \cdots \Delta X_{n}$ of $\beta$, each $X_{1}$ being an element of $B_{i}$, let $\omega^{1, \mu}$ be a chain mapping of $\gamma_{1}$ into $\mu$ defined as in Lemma 5 which is an extension of the zero-chain map $\omega_{\mu^{\prime}}$ defined to be $\sum \omega_{u}(v)$, where the summation extends over all vertices $u$ of $\mu$. That the map $\omega_{\mu^{\prime}}$ satisfies the hypothesis of Lemma 5 is shown by Lemma 4 and the fact that for the map $\omega_{i}$ as defined in Lemma 3 and for each one-simplex $A_{i}$ of $\alpha_{i}$ and maximal coherent subcomplex $X_{i}$ of $\beta_{i}$ each simplex of which has a nonzero coefficient in $\omega_{i}\left(A_{i}\right)$, the subchain of $\omega_{i} \delta\left(A_{i}\right)$ over those simplexes of $\beta_{i}$ which lie in $X_{i}$ bounds in $X_{i}$. Let $\omega^{1}$ be the map $\sum \omega^{1, \mu}$, where the summation extends over all $n$-product simplexes $\mu$ of $\beta$ of the form $X_{1} \Delta X_{2}$ $\Delta \cdots \Delta X_{n}$, each $X_{i}$ being an element of $B_{i} \cdot \omega^{1}$ is a chain mapping of the subcomplex $\rho_{1}$ of $\alpha$ into $\beta$.

The chain mapping $\omega$ will be constructed inductively. Suppose the chain map $\omega^{j}$ of the complex $\rho_{j}$ into $\beta$ is defined and has the following properties: $\omega^{j}$ is an extension of $\omega^{j-1}$ and for each $n$-product simplex $\gamma=A_{1} \Delta A_{2} \Delta \cdots \Delta A_{n}$ of $\rho_{j}$, if $B_{i}$ denotes the collection of all maximal coherent subcomplexes of $\beta_{i}$ each of whose simplexes has nonzero coefficients in $\omega_{i}\left(A_{i}\right)$, and for each complex $\mu=Y_{1} \Delta Y_{2} \Delta \cdots$ $\triangle Y_{n}$ of $\beta$, each $Y_{i}$ being an element of $B_{i}, \omega_{\mu}^{j}\left(\gamma^{i}\right)$, for each $i$-simplex $\gamma^{i}$ in $\gamma$, denotes the sum of those $i$-simplexes in $\mu$ with the same coefficients as they have in $\omega^{j}\left(\gamma^{i}\right)$, then $\omega_{\mu}^{j}$ is a chain mapping of $\gamma$ 
into $\mu$ which maps bounding zero-cycles into bounding zero-cycles, and $\omega^{i}\left(\gamma^{i}\right)=\sum \omega_{\mu}^{j}\left(\gamma^{i}\right)$, where the summation is over all $\mu$ of the form $Y_{1} \Delta Y_{2} \Delta \cdots \Delta Y_{n}$, each $Y_{i}$ being an element of $B_{i}$. Then $\omega^{j}$ can be extended to a chain mapping $\omega^{j+1}$ of the complex $\rho_{j+1}$ into $\beta$ in such a way that $\omega^{j+1}$ has the same properties for $\rho_{j+1}$ and $\beta$ as that given above for $\omega^{j}$ with respect to $\rho_{j}$ and $\beta$.

For the $n$-product simplex $\gamma_{j+1}=A_{1} \Delta A_{2} \Delta \cdots \Delta A_{n}$ of $\rho_{j+1}$, let $C_{i}$ be defined as was $B_{i}$ above but for the $A_{i}$ of $\gamma_{j+1}$. For each complex $\lambda=Z_{1} \Delta Z_{2} \Delta \cdots \Delta Z_{n}$ in $\beta$, each $Z_{i}$ being an element of $C_{i}$, and for each subsimplex $\gamma^{k}$ of an $n$-product proper subsimplex $\gamma$ of $\gamma_{j+1}$, let $\omega_{\lambda}\left(\gamma^{k}\right)=\sum \omega_{\mu}^{j}\left(\gamma^{k}\right)$, where the summation is over all $\mu$ of the form $Y_{1} \Delta Y_{2} \Delta \cdots \Delta Y_{n}$, each $Y_{i}$ being an element of $B_{i}$ and a subcomplex of $Z_{i}$. Since each $\omega_{\mu}^{j}$ satisfies the hypothesis of Lemma 5, $\omega_{\lambda}$ does; since $\lambda$ is zero-cyclic, $\omega_{\lambda}$ can be extended to a chain mapping $\omega_{\lambda}^{j+1}$ of $\gamma_{j+1}$ into $\lambda$. Define $\omega^{j+1}$ of $\gamma_{j+1}$ into $\beta$ to be $\sum \omega_{\lambda}^{j+1}$, where the summation is over all $\lambda$ of the form $Z_{1} \Delta Z_{2} \Delta \cdots \Delta Z_{n}$, each $Z_{i}$ being an element of $C_{i}$. For the $n$-product simplexes of $\rho_{j+1}$ other than $\gamma_{j+1}$, let $\omega^{j+1}$ be $\omega^{j}$. Each simplex of $\rho_{j+1}$ which lies in both $\gamma_{j+1}$ and another $n$-product simplex in $\rho_{j+1}$ also lies in an $n$-product simplex of $\rho_{j+1}$ common to both of them. Thus, the image of such a simplex under $\omega^{j}$ is the same as its image under $\omega^{j+1}$. It might be noted that each map $\omega_{\lambda}^{j+1}$ maps bounding zero-cycles into bounding zero-cycles, and so the chain mapping $\omega^{j+1}$ of $\rho_{j+1}$ into $\beta$ has the same properties for $\beta_{j+1}$ as has $\omega^{j}$ for $\rho_{j}$.

By mathematical induction there exists a chain mapping $\omega^{k}$ of $\rho_{k}$ into $\beta$ having similar properties. $\rho_{k}$ is $\alpha$. Denote $\omega^{k}$ by $\omega$. Since $\omega$ is an extension of $\omega^{\prime}$, it preserves the Kronecker index of zero-cycles. By its construction, $\omega$ has property (ii) of the conclusion of this theorem. Since $\pi$ also preserves Kronecker indices of zero-cycles, $\omega \pi$ does and $\omega \pi \sim 1$.

THEOREM 3. The Cartesian product of finitely many compact metric chainable continua is a quasi-complex.

Proof. Let $M_{1}, M_{2}, \cdots, M_{n}$ denote compact metric chainable continua. For each $i, 1 \leqq i \leqq n$, let $U_{1}^{\dagger}, U_{2}^{\dagger}, \cdots$ be a sequence of chains covering $M_{i}$ such that $U_{j+1}^{t}$ is a refinement of $U_{j}^{t}$ and $U_{j}^{t}$ is a $(1 / j)$-chain; let $\Phi_{j}^{i}$ denote the nerve of $U_{j}^{\imath}$. For $j<k$, let $\pi_{j}^{1, k}$ be a simplicial mapping of $\Phi_{k}^{i}$ into $\Phi_{j}^{i}$ induced by inclusion and let $\omega_{k}^{i, j}$ be the chain mapping of $\Phi_{j}^{i}$ into $\Phi_{k}^{i}$ as defined in Lemma 3. For each $j$, let $\Phi_{j}$ be $\Phi_{j}^{1} \Delta \Phi_{j}^{2} \Delta \cdots \Delta \Phi_{j}^{n}$ and define mappings $\pi_{j}^{k}$ of $\Phi_{k}$ into $\Phi_{j}$ and $\omega_{k}^{j}$ of $\Phi_{j}$ into $\Phi_{k}$ as in Lemma $6 . \Phi_{j}$ is the nerve of the covering of $M=M_{1} \times M_{2} \times \cdots \times M_{n}$ obtained by taking the Cartesian product 
of the coverings $U_{j}^{1}, U_{j}^{2}, \cdots, U_{j}^{n}$, and $\pi_{j}^{k}$ is a projection mapping of $\Phi_{k}$ onto $\Phi_{j}$. The proof that the continuum $M$ has Property B is just as that in Theorem 2.

The proof that $M$ has Property $\mathrm{C}$ is also much like that in Theorem 2. For each integer $i$, there is an integer $j$ such that if $G$ is a collection of sets in the open covering $U_{j}=U_{j}^{1} \times U_{j}^{2} \times \cdots \times U_{j}^{n}$ having a common point, some element of the covering $U_{i}=U_{i}^{1} \times U_{i}^{2} \times \cdots \times U_{i}^{n}$ contains the union of the elements of the collection $G$. If $U_{k}$ is a term of the sequence $U_{1}, U_{2}, \cdots$, let $U_{m}$ be the element of the set $\left\{U_{j}\right\} \cup\left\{U_{k}\right\}$ having the greater subscript. For any simplex $\sigma$ of $\Phi_{m}$, there is a simplex $\rho$ of $\Phi_{j}$ containing $\pi_{j}^{m}$ of $\sigma$ and all simplexes of $\Phi_{m}$ having nonzero coefficients in $\omega_{m}^{j} \pi_{j}^{m}(\sigma) . \pi_{i}^{j}(\sigma)$ is a vertex of $\Phi_{i}$. Hence, Property $\mathrm{C}$ is satisfied.

Comments. A conjecture suggested by the theorem that each compact metric continuum which is chainable has the fixed point property is that each compact metric continuum which has arbitrarily small "square-like" coverings ("cube-like," etc.) has the fixed point property. S. Eilenberg pointed out to the author that an example given by Borsuk [2] settles this conjecture in the negative for such continua with arbitrarily small "cube-like" coverings.

Another open question is to determine which of the compact plane continua not separating the plane are not zero-cyclic quasi-complexes. It would also be interesting to know if the Cartesian product of any two quasi-complexes is a quasi-complex.

Added in proof. The proofs of Theorems 1, 2 and 3, with only slight modifications, actually establish somewhat more general results. Let us enlarge the class of chainable continua to include those compact Hausdorff spaces having a cofinal collection $\omega$ of finite open coverings such that the elements of each member of $\omega$ can be linearly ordered in such a way that two of them intersect if and only if they are adjacent in the ordering. The proofs of Theorems 1, 2 and 3 hold for this class of spaces if instead of using arguments depending on a metric we use the following lemmas (see [6, pp. 19 and 326]):

If $X$ and $Y$ are compact Hausdorff spaces, then every finite open covering of $X \times Y$ has a refinement $\mathfrak{A} \times \mathfrak{B}$, where $\mathfrak{A}$ and $\mathfrak{B}$ are finite open coverings of $X$ and $Y$, respectively, and

If $f$ is a continuous map of the compact Hausdorff space $X$ into itself having no fixed point, there is a finite open covering $\mathfrak{A}$ of $X$ such that no star of $\mathfrak{A}$ meets its image under $f$.

T. R. Brahana has announced [7] the result that the direct product of two quasi-complexes is a quasi-complex. 


\section{BIBLIOGRAPHY}

1. R. H. Bing, Snake-like continua, Duke Math. J. vol. 18 (1951) pp. 653-663.

2. K. Borsuk, Sur un continu acyclique qui se laisse transformer topologiquement en lui même sans points invariants, Fund. Math. vol. 24 (1935) pp. 51-58.

3. S. Eilenberg and N. Steenrod, Foundations of algebraic topology, Princeton University Press, 1952.

4. O. H. Hamilton, $A$ fixed point theorem for pseudo-arcs and certain other metric continua, Proc. Amer. Math. Soc. vol. 2 (1951) pp. 173-174.

5. W. Hurewicz and H. Wallman, Dimension theory, Princeton University Press, 1948.

6. S. Lefschetz, Algebraic topology, Amer. Math. Soc. Colloquium Publications. vol. 27, New York, 1942.

7. T. R. Brahana, Products of quasi-complexes, Bull. Amer. Math. Soc. Abstract 62-1-110.

UNIVERSITY OF GEORGIA 